\title{
BOOLEAN ALGEBRAS WITH FEW ENDOMORPHISMS
}

\author{
SAHARON SHELAH
}

\begin{abstract}
Using diamond for $\kappa_{1}$ we construct a Boolean algebra in $\kappa_{1}$, whose only endomorphisms are those definable using finitely many elements and ultrafilters. We also generalize Rubin's construction to higher cardinals.
\end{abstract}

0. Introduction. The aim we state in the name of the paper can be interpreted in two ways: every endomorphism is "simply defined", or the number of endomorphisms is small. However for every ultrafilter $F$ on a Boolean algebra (or, equivalently maximal ideal $I=B-F$ ) we can define an endomorphism $T$ :

$$
T_{b}= \begin{cases}1, & b \in F \\ 0, & b \in I\end{cases}
$$

So "defined" should be interpreted as definable using maximal ideals. This is done in Theorem 1.4 (2) (saying there are indecomposable complicated Boolean algebras, see Definition 1.1, 2, 3) and Theorem 1.8(2) (saying any such algebra has only simply definable endomorphisms (see Definitions 1.5, 1.6)). Those theorems answer a question of Monk; for further historical background see $[\mathbf{R}]$.

As for the other interpretation (the number of endomorphisms should be small), Rubin [R] using $\diamond_{\kappa_{1}}$ builds a Boolean algebra of power $\kappa_{1}$, which we call here 1-Rubin (or Rubin). Such algebras in particular, have only $\aleph_{1}$ ideals and subalgebras. (The exact definition speaks on any set of $\aleph_{1}$ elements having some properties.) We generalize this to sets of $\aleph_{1} n$-tuples and get a Boolean algebra which is $n$-Rubin for each $n$ (Theorem 2.5). In fact we do this for $\lambda^{+}$instead $\aleph_{1}$, assuming $\diamond_{\lambda}$ and $\diamond_{\lambda^{+}}$, using [Sh1].

Variants of $\S 1$, in ZFC but for higher cardinals, will appear, as well as generalizing Theorem 2.5 to higher cardinals.

Further remarks. (1) We can generalize §1 using [Sh1], but it does not seem so interesting.

(2) We can ask in 2.5 for which Boolean algebras $C$ and set $K_{0}$ of $n$-tuples from $C, K_{0}=\left\{\left(b_{i}^{0}, \ldots, b_{i}^{n-1}\right): i<i_{0}<\lambda\right\}$ : for every set $K$ of $\lambda n$-tuples from $B\left(K_{0}, K_{1}\right.$ with disjoint sequences of distinct elements) we can find distinct $\left(a_{i}^{0}, \ldots, a_{i}^{n-1}\right) \in K\left(i<i_{0}\right)$ such that the mapping $a_{i}^{l} \mapsto b_{i}^{l}$ induces an embedding of the subalgebra of $C$ generated by $\left\{a_{i}^{l}: l<n, i<i_{0}\right\}$ into $B$. For $n=1, \lambda=\aleph_{1}$ Rubin [R] answers this; for $n=1, \lambda>\aleph_{1}$, the conditions

Received by the editors November 18, 1977.

AMS (MOS) subject classifications (1970). Primary 02J05; Secondary 02H15.

Key words and phrases. Boolean algebra, endomorphism.

(C) 1979 American Mathematical Society 0002-9939/79/0000-0174/\$03.00 
are essentially the same. (No union of members of $K_{0}$ is 1 , no intersection of members of $K_{0}$ is empty, and they can be well ordered so that no one is a Boolean combination of previous ones.) For $n>0$, there seems to be no essential problem but it seems hard to phrase it intelligibly.

(3) It is open whether there are $n$-Rubin not $(n+1)$-Rubin Boolean algebras (in particular this is hopeful for $n=1, \lambda=\kappa_{1}$ ).

The results from $[\mathbf{R}]$ generalize easily from the case $\lambda=N$ to other cases of $\$ 2$ at least for $\lambda=\mu^{+}, B \mu$-saturated, but the other questions were not checked (can $\mu^{+}<\lambda$, can $\lambda$ be singular, etc.).

Notation. Let $m, n, k, l$ denote natural numbers, $\alpha, \beta, \gamma, i$ ordinals, $\lambda$ an infinite cardinality. $P(A)$ is the family of subsets of $A$.

Let $B$ denote a Boolean algebra, $0,1, \cap, \cup,-, \leqslant$ operations and relations of Boolean algebras.

We let $[a, x]^{\mathrm{if}(t)}$ be $a \subseteq x$ if $t$ is true and $a \cap x=0$ otherwise.

\section{Complicated Boolean algebras and Boolean algebras with only definable} endomorphisms.

1.1 Definition. A Boolean algebra $B$ is called complicated if for any candidate $\left\{\left(a_{n}, b_{n}\right): n<\omega\right\}$ for $B$ (which means: $m \neq n$ implies $a_{n} \cap a_{m}=0$, $b_{n} \cap b_{m}=0$ and for each $n, b_{n} \underline{\ell} a_{n}$ and of course $\left.a_{n}, b_{n} \in B\right)$ there is a witness set $S \subseteq \omega$ i.e. such that

(i) for some $x \in B$ for every $n\left[a_{n}, x\right]^{\mathrm{if}(n \in S)}$,

(ii) for no $x \in B$ for every $n\left[b_{n}, x\right]^{\mathrm{ji}(n \in S)}$.

1.2 Definition. An ideal $I$ of a Boolean algebra $B$ is called indecomposable if there are no nonprincipal disjoint ideals $I_{0}, I_{1}$ such that $I$ is generated by them (so $I_{0}, I_{1} \subseteq I$ ). Clearly such filter is not principal (otherwise $I$ is called decomposable). ( $I$ is principal if for some $a, I=\{b \in B: b \leqslant a\}$.)

1.3 Definimion. A Boolean algebra $B$ is called indecomposable if every nonprincipal maximal ideal of $B$ is indecomposable.

1.4. THEOREM (1) (CH) There is an atomless complicated Boolean algebra of cardinality $\aleph_{1}$.

(2) $\left(\diamond_{\kappa_{1}}\right)$ There is an atomless indecomposable complicated Boolean algebra of cardinality $\mathrm{N}_{1}$.

(3) In (1) we can assume that any maximal infinite antichain and chain in $B$ is uncountable. In (2) we can assume this or that there are no uncountable chains and antichains in $B$.

REMARK. (1) Instead of $\mathrm{CH}$ it suffices to assume in (1) that the union of $<2^{\kappa_{0}}$ many nowhere dense subsets of $P(\omega)$ is not $P(\omega)$.

(2) We may want $B$ to be atomic with $\kappa_{0}\left(\kappa_{1}\right)$ atoms. For this always enlarge $\left\{a_{n}^{\alpha}: n<\omega\right\}$ to a maximal antichain $\left\{a_{\beta}^{\alpha}: \beta<\beta_{\alpha}\right\}$ in the following proof.

Proof. (1) We define by induction on $\alpha<\aleph_{1}$ an atomless Boolean algebra $B_{\alpha}$ and types $p_{\beta}(\beta<\alpha)$ such that: 
(a) $B_{\alpha}\left(\alpha<\omega_{1}\right)$ is increasing and continuous, each $B_{\alpha}$ is countable and for convenience its set of elements is $\omega(1+\alpha)$.

(b) $p_{\alpha}=\left\{\left[b_{n}^{\alpha}, x\right]^{\mathrm{if}(n \in S(\alpha))}: n<\omega\right\}$ where $b_{n}^{\alpha} \in B_{\alpha}, b_{n}^{\alpha} \neq 0, n \neq m \Rightarrow b_{n}^{\alpha} \cap$ $b_{m}^{\alpha}=0$ and $S(\alpha) \subseteq \omega$ and $S(\alpha), \omega-S(\alpha)$ are infinite.

(c) No element of $B_{\alpha}$ realizes any $p_{\beta}(\beta \leqslant \alpha)$.

Our desired algebra will be $B=\cup_{\alpha<\omega_{1}} B_{\alpha}$.

As we use the continuum hypothesis, by standard methods we can assume that for each $\alpha$ we are given a candidate $\left\{\left(a_{n}^{\alpha}, b_{n}^{\alpha}\right): n<\omega\right\}$ for $B_{\alpha}$, so that every candidate for $B$ appears in the list.

Now it suffices to define $B_{\alpha+1}, S(\alpha)$, assuming $B_{\alpha}, p_{\beta}(\beta<\alpha)$ are given such that $S(\alpha)$ is a witness for the candidate $\left\{\left(a_{n}^{\alpha}, b_{n}^{\alpha}\right): n<\omega\right\}$ in $B_{\alpha+1}$, and $B_{\alpha+1}$ does not realize $p_{\beta}(\beta \leqslant \alpha)$. Notice $S(\alpha)$ defines $p_{\alpha}$ (see (b)), and that for $\alpha$ zero or limit, there is no problem to define $B_{\alpha}$.

After the choice of $S(\alpha)$ we will define $B_{\alpha+1}$ as follows: it is generated by $B_{\alpha}$ and $x_{\alpha}$, freely except that $x_{\alpha}$ realizes $\left\{\left[a_{n}^{\alpha}, x\right]^{\mathrm{ji}(n \in S(\alpha))}: n<\omega\right\}$.

Now all demands on $B_{\alpha+1}$ are translated to demands on $S(\alpha)$. Now we should show that they are compatible; for this we shall show there are countably many demands (remembering $\alpha<\kappa_{1}$ ) each of them being: $S(\alpha)$ lies outside a subset of $P(\alpha)$ which is of the first category (or even is nowhere dense). By the Baire category theorem this is sufficient.

So let $\bar{\sigma}=\left(\sigma_{1}, \sigma_{2}\right)$ denote a pair of disjoint finite subsets of $\omega, V_{\bar{\sigma}}=\{S \subseteq$ $\left.\omega: \sigma_{1} \subseteq S, \sigma_{2} \cap S=\varnothing\right\}$. Let $\bar{\sigma} \subseteq \bar{\sigma}^{\prime}$ mean $\sigma_{1} \subseteq \sigma_{1}^{\prime}, \sigma_{2} \subseteq \sigma_{2}^{\prime}$ hence $V_{\bar{\sigma}^{\prime}} \subseteq V_{\bar{\sigma}}$. So let us deal with the demand " $B_{\alpha+1}$ does not realize $p_{\beta}$ " where $\beta \leqslant \alpha$. So for each partition $b_{1}, b_{2}, b_{3}, b_{4}$ of 1 in $B_{\alpha}$, and $\bar{\sigma}$ we have to find $\bar{\sigma}^{\prime}, \bar{\sigma} \subseteq \bar{\sigma}^{\prime}$ which ensure that $y=b_{1} \cup\left(b_{2} \cap x\right) \cup\left(b_{3}-x\right)$ does not realize $p_{\beta}$. (As there are countably many $\beta$ 's and such partitions, this is sufficient.) (Note $y$ is a "general" element of $B_{\alpha+1}$.)

By inessential changes we can assure $\sigma_{1} \cup \sigma_{2}=\{0, \ldots, n-1\}$ and $a_{0}^{\alpha}$ $\cup \cdots \cup a_{n-1}^{\alpha} \subseteq b_{1} \cup b_{4}$.

First assume $\beta<\alpha$. If for some $m \geqslant n$ and $k, b_{k}^{\beta} \cap a_{m}^{\alpha} \neq 0$ then we can assure $y$ does not realize $p_{\beta}$, by assuring not $\left[b_{k}^{\beta}, y\right]^{\mathrm{if}(m \in S(\beta))}$ and this we do by adding $m$ to $\sigma_{1}$ or $\sigma_{2}$ (and so getting $\bar{\sigma}^{\prime}$ ). If there is no such $k$, but for some $k$, $b_{k}^{\beta}-b_{1}-b_{4} \neq 0$, the freeness of $x_{\alpha}$ assures our desire. In the remaining case, if $y$ realizes $p_{\beta}$ (for some choice of $S(\alpha) \in V_{\bar{\sigma}}$ ) then $b_{1}$ will do as well, contradiction.

So remains the case $\beta=\alpha$. If for some $m \geqslant n, b_{1} \cap b_{m}^{\alpha} \neq 0$ we adjoin $m$ to $\sigma_{2}$ (to get $\bar{\sigma}^{\prime}$ ), and if for some $m \geqslant n, b_{4} \cap b_{m}^{\alpha} \neq 0$ we adjoin $m$ to $\sigma_{1}$, and this is clearly sufficient. If for some $m \neq l \geqslant n,\left(b_{m}-a_{m}\right) \cap a_{l} \neq 0$ we adjoin $m$ to $\sigma_{1}$ and $l$ to $\sigma_{2}$. In the remaining case the freeness of $x_{2}$ ensures the result.

Now it is trivial to check $B=\cup_{\alpha<\omega_{1}} B_{\alpha}$ is as required.

(2) We repeat the proof of the first part: but the enumeration of candidates is only for successor ordinals and for such $\alpha$ 's we act as before. (Also for limit $\beta$ we have two $p_{\beta}$ 's and they are defined a little differently.) For limit $\alpha<\omega_{1}$, 
we are given $I_{0}^{\alpha}, I_{1}^{\alpha} \subseteq B_{\alpha}$, such that eventually for every $I_{0}, I_{1} \subseteq B,\{\alpha$ : $\left.I_{1}^{\alpha}=I_{1} \cap B_{\alpha}, I_{0}^{\alpha}=I_{0}^{\alpha} \cap B_{\alpha}\right\}$ is a stationary set of limit ordinals (hence nonempty). So if $I_{0}, I_{1}$ were disjoint nonprincipal ideals in $B$, whose union generates a maximal ideal in $B$, so are $I_{1} \cap B_{\alpha}, I_{0} \cap B_{\alpha}$ in $B_{\alpha}$ for a closed unbounded set of $\alpha$ 's. So some $\alpha$ satisfies both demands, so it suffices in defining $B_{\alpha+1}, p_{\alpha}$ to assure no such $I_{0}, I_{1}$ will exist.

So suppose $I_{0}^{\alpha}, I_{1}^{\alpha}$ are disjoint nonprincipal ideals in $B_{\alpha}$ whose union generates a maximal ideal. We can find pairwise disjoint $a_{n}^{\alpha} \in I_{0}^{\alpha} \cup I_{1}^{\alpha}$, such that $\left\{a_{2 n}: n<\omega\right\},\left\{a_{2 n+1}: n<\omega\right\}$ generate $I_{0}^{\alpha}, I_{1}^{\alpha}$ resp. We shall choose $S(\alpha) \subseteq \omega$, so that there are infinitely many odds and even, in $S(\alpha)$ and in its complement, and define $B_{\alpha+1}$ as in (1).

We now let for $l=0,1, p_{\beta}^{l}=\left\{\left[a_{n}, x\right]^{\mathrm{iff}[n \in S(\alpha)]}: n=l \bmod 2\right\}$. We have to define $S(\alpha)$ so that again all types up to and including this stage are omitted, and this is done in the same way (using the properties of $I_{0}^{\alpha}, I_{1}^{\alpha}$ ).

(3) is left to the reader, we may have to change the last phrase of (b). (To make $B_{\alpha}$ atomless we may sometimes add freely an $x$.)

1.5 Definition (1) A scheme of an endomorphism of $B$ consists of a partition $a_{0}, a_{1}, b_{2}, \ldots, b_{n-1}, c_{0}, \ldots, c_{m-1}$ of 1 , maximal nonprincipal ideal $I_{l}$ below $b_{l}$ for $l<n$, nonprincipal disjoint ideals $I_{l}^{0}, I_{l}^{1}$ below $c_{l}$ which generates a maximal ideal below $c_{l}$ for $l<m$, a number $k<n$, and a partition $b_{0}^{*}, \ldots, b_{n-1}^{*}, c_{0}^{*}, \ldots, c_{m-1}^{*}$ of $a_{0} \cup b_{0} \cup \cdots \cup b_{k-1}$. We assume also that $k+m>0 \Rightarrow a_{0}=0,(n-k)+m=0 \Rightarrow a_{1}=0$ and except in those cases there are no zero elements in the partition.

(2) the scheme is simple if $m=0$.

(3) The endomorphism of the scheme is the unique endomorphism (see 1.7)

$T: B \rightarrow B$ such that:

(i) $T x=0$ when $x \leqslant a_{0}$ or $x \in I_{l}, l<k$, or $x \in I_{l}^{0}, l<m$.

(ii) $T x=x$ when $x \leqslant a_{1}$ or $x \in I_{l}, k \leqslant l<n$ or $x \in I_{l}^{1}, l<m$.

(iii) $T\left(b_{l}\right)=b_{l}^{*}$ when $l<k$.

(iv) $T\left(b_{l}\right)=b_{l} \cup b_{l}^{*}$ when $k \leqslant l<n$.

(v) $T\left(c_{l}\right)=c_{l} \cup c_{l}^{*}$ when $l<m$.

1.6 Definition. An endomorphism of the Boolean algebra $B$ is (simply) definable if there is a (simple) scheme which defines it.

1.7 Claim. Any scheme of an endomorphism of $B$ defines uniquely an endomorphism of $B$.

REMARK. The representation is almost unique. We can interchange elements among $\bigcup_{l<k} I_{l} \cup \cup_{l<m} I_{l}^{0}$ and among $\bigcup_{l \geqslant k} I_{l} \cup \cup_{l<m} I_{l}^{1}$.

Proof. Easy.

1.8 THEOREM. (1) Every endomorphism of a complicated Boolean algebra is definable.

(2) If in addition the Boolean algebra is indecomposable, then every endomorphism is simply definable. 
Proof. Part (2) follows easily from (1) and Definition 1.3 (for if $I_{l}^{0}, I_{l}^{1}$ are as described in Definition 1.5 , adjoin $1-c_{l}$ to $I_{l}^{1}$, and get a contradiction to the indecomposability of $B$ ). So let us prove (1).

So let $T: B \rightarrow B$ be an endomorphism.

Stage (i). Define

$$
\begin{aligned}
& I_{0}=\{b \in B: \text { for every } a \leqslant b, T a=0\}, \\
& I_{1}=\{b \in B: \text { for every } a \leqslant b, T a=a\} .
\end{aligned}
$$

Note that as $a \leqslant b \Rightarrow T a \leqslant T b$, clearly $T a=0 \Rightarrow a \in I_{0}$, but clearly not necessarily $T a=a \Rightarrow a \in I_{1}$.

Clearly $I_{0}, I_{1}$ are disjoint ideals of $B$, and $I$ is the ideal they generate.

Stage (ii). In $B$ there are no disjoint elements $a_{n}(n<\omega)$ such that $T a_{n} \nless a_{n}$.

Clearly $m \neq n \Rightarrow T a_{m} \cap T a_{n}=0$ (as it is $T\left(a_{m} \cap a_{n}\right)=T(0)=0$ ), so $\left\{\left(a_{n}, T a_{n}\right): n<\omega\right\}$ is a candidate (see Definition 1.1), so (as $B$ is complicated) there is $S \subseteq \omega$ and $c \in B$ realizing $\left\{\left[a_{n}, x\right]^{\mathrm{if}(n \in S)}: n \leqslant \omega\right\}$, but no $c^{\prime} \in b$ realizes $\left\{\left[T a_{n}, x\right]^{\mathrm{if}(n \in S)}: n<\omega\right\}$, but $T c$ realizes it, contradiction.

Stage (iii). If $b \in B$, and $c \leqslant b \Rightarrow T c \leqslant c$ then $b \in I$.

Clearly for every $c \leqslant b, T(c-T c) \leqslant c-T c$ by the hypothesis of the stage, and $T(c-T c) \leqslant T c$ as $c-T c \leqslant c$, hence $T(c-T c)=0$. We can conclude that for $c \leqslant b, c-T c \in I_{0}$, and $T c=T(T c)$.

Let $c_{0}=T b$, so $c_{0} \leqslant b, T c_{0}=c_{0}$ and suppose $c \leqslant c_{0}, T c \neq c$. Then $T c<c$, and let $d=c-T c \neq 0$ (so $\left.d \leqslant c \leqslant c_{0}, d \in I_{0}\right)$ now $T c_{0}=T\left(\left(c_{0}-\right.\right.$ d) $\cup d)=T\left(c_{0}-d\right) \cup T(d)=T\left(c_{0}-d\right) \cup 0=T\left(c_{0}-d\right) \leqslant c_{0}-d$ so $T c_{0}<c_{0}($ as $d \neq 0)$ but $T c_{0}=c_{0}$ as $c_{0}=T b$, contradiction. So $c \leqslant c_{0} \Rightarrow T c$ $=c$, i.e. $c_{0} \in I_{1}$, but $b=(b-T b) \cup T b=(b-T b) \cup c_{0}, b-T b \in I_{0}, c_{0}$ $\in I_{1}$, so $b \in I$, as desired.

Stage (iv). $B / I$ is finite.

Otherwise there are pairwise disjoint nonzero $a_{n} / I(n<\omega)$. By replacing $a_{n}$ by $a_{n}-\cup_{l<n} a_{l}$, we can assure the $a_{n}$ 's are pairwise disjoint, and of course $a_{n} \notin I$. If for every $n$ there is $a_{n}^{\prime} \leqslant a_{n}, T a_{n}^{\prime} \nless a_{n}^{\prime}$, we get a contradiction by (ii), but if $c \leqslant a_{n} \Rightarrow T c \leqslant c$ then by (iii) $a_{n} \in I$, again a contradiction. So necessarily $B / I$ is finite.

Stage (v). $T$ is definable.

Let $b_{l} / I(l<n)$ be the atoms of $B / I$, and w.l.o.g. $b_{l}(l<n)$ is a partition of 1 . Clearly the restriction of $I$ to each $b_{l}$ is a maximal filter. The rest is easy checking.

\section{On Rubin Boolean algebras.}

2.1 Definition. (1) Let $B$ be a Boolean algebra. A formal $n$-interval is: a partition $b_{0}, \ldots, b_{m-1}$ of 1 in $B, b_{k} \neq 0$ and $\tau_{k}^{l} \in\left\{0, b_{k}, x_{k}, b_{l}-x_{k}\right\}(l<n$, $k<m$ ) and elements $c_{k}^{0}$, $c_{k}^{1}$, where $0 \leqslant c_{k}^{0}<c_{k}^{1} \leqslant b_{k}$ (for not necessarily atomless $B$ we may want to demand $c_{k}^{1}-c_{k}^{0}$ is infinite). We name a formal 
$n$-interval $\nu$, and then write $n_{\nu}, m_{\nu}, b_{l}^{\nu}, \dot{\tau}_{k}^{\nu, l}, c_{k}^{\nu, l}$ when the identity of $\nu$ is not clear.

(2) The formal $n$-interval is simple if $c_{k}^{0}=0, c_{k}^{1}=b_{k}$. We say $\nu$ is a formal $n$-subinterval of $\nu^{*}$ if $c_{k}^{\nu^{*}, 0} \leqslant c_{k}^{\nu, 0}<c_{k}^{\nu, 1} \leqslant c_{k}^{\nu^{*}, 1}$ and $b_{l}^{\nu}=b_{l}^{\nu^{*}}, \tau_{k}^{\nu, l}=\tau_{k}^{\nu^{*}, l}$.

(3) Let $\varphi^{\nu}(\bar{x}, \bar{y})$ be $\bigwedge_{l<n ; k<m} y_{l} \cap b_{k}=\tau_{k}^{l}\left(\wedge \bigwedge_{k} c_{k}^{0} \leqslant x_{k} \leqslant c_{k}^{l}\right)$ and $\psi^{\nu}(\bar{y})$ $=(\exists \bar{x}) \varphi^{\nu}(\bar{x}, \bar{y})$.

(4) We say $\bar{a}=\left\langle a_{0}, \ldots, a_{n-1}\right\rangle$ realizes $\nu$ if $\varphi^{\nu}(\bar{a})$ is satisfied.

We now consider a generalization. Let $\lambda, \mu$ be fixed infinite cardinals in this section.

2.2 Definition. (1) Let $B$ be a Boolean algebra, $\gamma$ an ordinal $<\mu$. A formal $\gamma$-interval is: nonzero elements $b_{i}\left(i<i_{1}\right), i_{0}<i_{1}$, and ordinals $i^{\alpha}(\alpha<\gamma)$ such that $\Sigma_{\alpha<\gamma} i^{\alpha}<\mu, i<j<i_{0} \Rightarrow b_{i} \cap b_{j}=0, i<i_{0} \leqslant j<i_{1} \Rightarrow\left[b_{i} \subseteq b_{j}\right.$ or $\left.b_{i} \cap b_{j}=0\right]$, terms $\tau_{i}^{\alpha}\left(\alpha<\gamma, i<i_{0}\right)$ such that for $i<i_{0}, \tau_{i}^{\alpha} \in\left\{0, b_{i}, x_{i}, b_{i}\right.$ $\left.-x_{i}\right\}$ and for $i_{0} \leqslant i<i_{1}$, formulas $\sigma_{i}$ of the form: the intersection of $b_{i}$ with finitely many $y_{\alpha}$ 's, $\left(1-y_{\alpha}\right)$ 's is empty (but no two have the same form) and we let for $i<i_{0}, \sigma_{i}^{\alpha}=\left[y_{\alpha} \cap b_{i}=\tau_{i}^{\alpha}\right] \wedge 0<x_{i}<b_{i}$ and the set of $\sigma$ 's is finitely satisfiable in $B$.

We name formal $\gamma$-intervals by $\nu$, and then write $\gamma^{\nu}$ instead of $\gamma, i_{1}^{\nu}, b_{i}^{\nu}$, etc. When the identity of $\nu$ is clear we omit it.

(2) If $\gamma=1$ we can assume $i_{1}=i_{0}+2, \tau_{i}=x_{i}$ for $i<i_{0}$, and all $b_{i}$ 's are pairwise disjoint and $\sigma_{i_{0}}=\left[y_{0} \cap b_{i_{0}}=0\right], \sigma_{i_{1}}=\left[\left(1-y_{0}\right) \cap b_{i_{0}+1}=0\right]$.

(3) We say $\nu$ is a formal $\gamma$-subinterval of $\nu^{*}$ if $\gamma^{\nu}=\gamma^{\nu^{*}}, i_{0}^{\nu}=i_{0}^{\nu^{*}}, \tau_{i}^{\nu, \alpha}=$ $\tau_{i}^{\nu^{*}, \alpha}, b_{i}^{\nu} \leqslant b_{i}^{\nu^{*}}$ for $i<i_{0}, \sigma_{i}^{\nu}=\sigma_{i}^{\nu^{*}}$ when $i_{0}^{\nu} \leqslant i<i_{1}^{\nu^{*}}, i_{1}^{\nu^{*}} \leqslant i_{1}^{\nu}$. If $n=1$, by assumption, we can demand $i_{0}^{\nu^{*}}=i_{0}^{\nu}, i_{1}^{\nu}=i_{1}^{\nu^{*}}$, and $b_{i}^{\nu} \leqslant b_{i}^{\nu^{*}}$ for $i<i_{0}$, $b_{i_{0}}^{\nu} \geqslant b_{i_{0}}^{\nu^{*}}, b_{i_{0}+1}^{\nu} \geqslant b_{i_{0}+1}^{\nu^{*}}$ and $b_{i}^{\nu^{*}} \leqslant\left(b_{i}^{\nu} \cup b_{i_{0}}^{\nu} \cup b_{i_{0}+1}^{\nu}\right)$ for $i<i_{0}$.

(4) Let $\psi^{\nu}(\bar{x}, \bar{y})$ be the conjunction of the formulas $\tau_{i}^{\alpha}=y_{\alpha} \cap b_{i}\left(i<i_{0}\right)$, $\sigma_{i}^{\alpha}\left(i_{0} \leqslant i<i_{1}\right)\left(\alpha<\gamma_{0}\right)$.

Let $\varphi^{\nu}(\bar{y})=(\exists \bar{x}) \psi^{\nu}(\bar{x}, \bar{y})$.

(5) We say $\bar{a}=\left\langle a_{\alpha}: \alpha\langle\gamma\rangle\right.$ realizes $\nu$ if $\varphi^{\nu}(\bar{a})$ holds.

Notation. $K$ will be a family of sequences of a fixed length from a Boolean algebra.

2.3 Definition. Let $B$ be a Boolean algebra, $\gamma<\mu$ and $K$ a family of $\gamma$-tuples of elements of $B$. We call $B$-small $(\lambda$-small) if for any formal $\gamma$-interval there is a formal $\gamma$-subinterval such that no (such that $<\lambda$ ) $\gamma$-tuple from $K$ realizes it.

2.4 Definition. (1) An atomless Boolean algebra $B$ of power $\geqslant \lambda$ is called $\gamma$-Rubin $\left[\gamma-{ }^{*}\right.$ Rubin] $(\gamma<\lambda)$, if any $\lambda$-small (any small) family of disjoint $\gamma$-tuples from $B$ has power $<\lambda$. We demand also that below each nonzero element there are $\lambda$ elements (otherwise there are $<\lambda$ exceptions, so this is a technical demand).

(2) If $n=1$ we omit it, and if it holds for every $\gamma<\mu$ we omit it and write strongly.

So Rubin [R] proves (assuming $\diamond_{\kappa_{1}}$, when $\lambda=\kappa_{1}, \mu=\kappa_{0}$ ) the existence of a 
Rubin Boolean algebra of cardinality $\aleph_{1}$. The demand $\lambda$-small instead of small is equivalent.

2.5 TheOREM. Let $\lambda=\mu^{+}, S=\{\alpha<\lambda$ : cf $\alpha=\mu\}$, $\mu$ regular and assume $\diamond_{\mu}$, hence $\mu=\mu^{<\mu}$ and $\diamond_{s}$ holds. There is a strongly Rubin Boolean algebra which is $\mu$-saturated.

REMARK. The proof gives a little more then $\mu$-saturation.

Proof. We use the construction of [Sh1] on omitting types in $L(Q)$. Our theory is the first-order theory of atomless Boolean algebras together with the axiom $(\forall x)[x \neq 0 \rightarrow(Q y) y<x]$, and it is easy to check it is complete and has elimination of quantifiers.

Now we build by induction on $\alpha$ atomless Boolean algebras $B_{\alpha}(\alpha<\lambda)$ and $\gamma_{\beta}$-types $p_{\beta}(\beta<\alpha)$ over $B_{\beta}$ such that

(1) $B_{\alpha}(\alpha<\lambda)$ is increasing and continuous, each $B_{\alpha+1}$ is saturated of cardinality $\mu$, and for convenience its set of elements is $\mu(1+\alpha)$.

(2) $p_{\beta}$ is an $\gamma_{\beta}$-type over $B_{\beta}\left(\gamma_{\beta}<\mu\right)$ which has no support (see [Sh1]) over each $B_{\alpha}, \alpha \geqslant \beta$, hence each $B_{\alpha}$ does not realize it.

By [Sh1] we just have to define for each given $B_{\alpha}$ a type $p_{\beta}$ with parameters over $B_{\alpha}$ which has no support over it. Now by $\diamond_{S}$ for each $\alpha \in S$ we will be given a $\gamma_{\alpha}<\mu$ and a family $K_{\alpha}$ of $\gamma_{\alpha}$-tuples of elements from $B_{\alpha}$, such that for each $\gamma<\mu$ and family $K$ of $\gamma$-tuples from $B=\cup_{\alpha<\gamma} B_{\alpha},\left\{\alpha: K \cap B_{\alpha}^{\gamma}=\right.$ $\left.K_{\alpha}\right\}$ is stationary. Note that for each such small $K,\left\{\alpha \in S: K \cap B_{\alpha}^{\gamma}\right.$ is small and for each formal $\gamma$-interval $\nu$ over $B_{\alpha}, \nu$ is realized in $K_{\alpha}$ iff it is realized in $K$, and is realized by $\lambda$ members of $K$ iff for every $\beta<\alpha,\left(B_{\alpha}-B_{\beta+1}\right)^{\gamma} \cap K$ $\neq 0\}$ is a closed unbounded set of $\alpha$ 's, hence for each such $K$ there is an $\alpha \in S$ for which $K \cap B_{\alpha}^{\gamma}$ is small and $K \cap B_{\alpha}^{\gamma}=K_{\alpha}, \gamma=\gamma_{\alpha}$. Now we shall try to let

$$
\begin{aligned}
p_{\alpha}=\left\{\neg \varphi^{\nu}(\bar{y}): \text { no } \gamma_{\alpha} \text {-tuple from } K \text { realizes } \nu,\right. \\
\text { a simple formal } \left.n_{\alpha} \text {-interval from } B_{\alpha}\right\} \\
\cup\left\{\bigvee_{i<\gamma_{\alpha}} x_{i} \neq a_{i}: a_{0}, \ldots \in B_{\alpha}\right\}
\end{aligned}
$$

(notice $p_{\alpha}$ consists of negations of conjunctions of first order formulas) by the $\mu$-saturation we can get rid of the $\exists \bar{x}$. If $p_{\alpha}$ is not realized in $B$, we see that no $n$-tuple from $K$ is disjoint from $B_{\alpha}$, hence $K$ has cardinality $\leqslant \mu$ or is not disjoint so we shall finish. We only have to prove $p_{\alpha}$ has no support over $B_{\alpha}$. So suppose

$$
Q x_{1} \exists x_{1}^{\prime} Q x_{2} \exists x_{2}^{\prime} \ldots \exists y_{0}, \ldots, y_{\gamma-1} \varphi\left(x_{1}, \ldots, y_{0}, \ldots, y_{\gamma-1}, b_{0}, \ldots\right)
$$

is a support $\left(b_{0}, \ldots \in B_{\alpha},|\varphi|<\mu\right)$. We can represent $\varphi$ as a disjunction of conjunctions each conjunction containing complete information as to which Boolean combination of the $x$ 's, $y$ 's and $b$ 's is empty. We can then replace it 
w.l.o.g. by one of the disjunctions. Then by using more parameters, we can add information to $\varphi$, so it is still a support, but $\varphi$ is of the form $\psi^{v}$ for some $\nu$, so we finish.

ADDED IN PROOF. 1. Monk improved 1.4(1) by getting a complicated Boolean algebra of power $2^{\mathrm{N}_{0}}$ without assuming $\mathrm{CH}$.

2. It seems that the authors proved 1.4(2) assuming $\mathrm{CH}$ only.

\section{REFERENCES}

[R] M. Rubin, On Boolean algebra with few endomorphisms, Trans. Amer. Math. Soc. (to appear).

[Sh1] S. Shelah, Models with second order properties. III, Omitting types for $L(Q)$ in higher cardinals, Proc. Sympos. Model Theory (West Berlin 1977), Edited by K. Makowski, Arch. Math. Logik Grundlagenforsch.

Institute of Mathematics, The Hebrew University, Jerusalem, IsRael (Current address)

II. Mathematisches Institut, Freie Universttät, Berlin, West Germany 